\title{
Erratum to: Prognostic factors for brain metastases from non-small cell lung cancer with $E G F R$ mutation: influence of stable extracranial disease and erlotinib therapy
}

\author{
Akimasa Sekine $^{1} \cdot$ Hiroaki Satoh $^{2} \cdot$ Tae Iwasawa $^{3} \cdot$ Katsumi Tamura $^{4}$. \\ Kenji Hayashihara $^{5}$ - Takefumi Saito ${ }^{5}$. Terufumi Kato ${ }^{1} \cdot$ Mito Arai $^{6}$. \\ Koji Okudela $^{7} \cdot$ Kenichi Ohashi $^{7} \cdot$ Takashi Ogura $^{1}$
}

Published online: 23 April 2015

(c) Springer Science+Business Media New York 2015

\section{Erratum to: Med Oncol (2014) 31:228 \\ DOI 10.1007/s12032-014-0228-9}

The original version of this article unfortunately contained a mistake. Table 3 was published with incorrect values. The corrected Table 3 is given below. The authors regret the inconvenience caused to the readers in this regard.

The online version of the original article can be found under doi:10.1007/s12032-014-0228-9.

Akimasa Sekine

Akimasa.Sekine@gmail.com

1 Department of Respiratory Medicine, Kanagawa

Cardiovascular and Respiratory Center, Tomioka-Higashi

6-16-1, Kanazawa-ku, Yokohama, Japan

2 Department of Internal Medicine, Mito Medical Center, University of Tsukuba, Mito, Japan

3 Department of Radiology, Kanagawa Cardiovascular and Respiratory Center, Yokohama, Japan

4 Department of Radiology, National Hospital Organization, Ibaraki-Higashi National Hospital, Ibaraki, Japan

5 Department of Respiratory Medicine, National Hospital Organization, Ibaraki-Higashi National Hospital, Ibaraki, Japan

6 Department of Radiology, Graduate School of Medicine, Yokohama City University, Yokohama, Japan

7 Department of Pathology, Graduate School of Medicine, Yokohama City University, Yokohama, Japan 
Table 3 Treatment history after brain metastases diagnosis
EGFR mutation status

EGFR mutant (89)

21

Both WBRT and radiosurgery

EGFR-TKIs
Yes/no

Gefitinib

Erlotinib

Both gefitinib and erlotinib
$5 / 84$

$57 / 32$

28

8

$5 / 103$

$87 / 21$

28

43

16

$66 / 23$

$26 / 82$

$<0.0001 *$

$0.010^{*}$
8

15

12

20

$p$ value

Wild-type (108)

0.76

$W B R T$ whole brain radiotherapy

$* p<0.05$ 